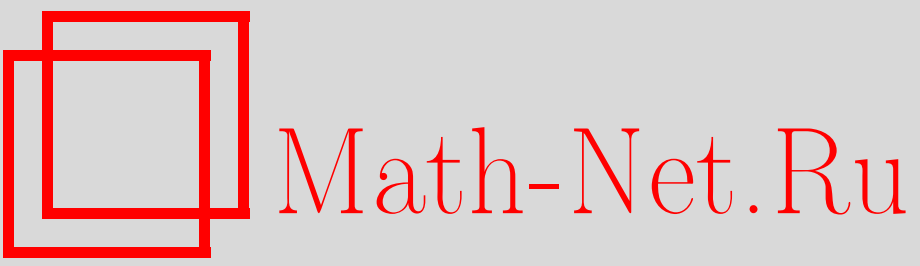

Н. А. Колодий, Об измеримости по параметру стохастического интеграла, управляемого двупараметрическим сильным мартингалом, Теория вероятн. и ее примен., 2011, том 56, выпуск 1, 159-167

DOI: https://doi.org/10.4213/tvp4332

Использование Общероссийского математического портала Math-Net.Ru подразумевает, что вы прочитали и согласны с пользовательским соглашением

http: //www . mathnet.ru/rus/agreement

Параметры загрузки:

IP: 54.92 .164 .108

26 апреля 2023 г., 15:24:33 


\title{
ОБ ИЗМЕРИМОСТИ ПО ПАРАМЕТРУ СТОХАСТИЧЕСКОГО ИНТЕГРАЛА, УПРАВЛЯЕМОГО ДВУПАРАМЕТРИЧЕСКИМ СИЛЬНЫМ МАРТИНГАЛОМ
}

\begin{abstract}
Приведены достаточные условия измеримости по параметру предела последовательности случайных полей с траекториями в $\mathbb{D}$. Доказана измеримость по параметру квадратической вариации двупараметрического сильного мартингала. Получены достаточные условия измеримости по параметру стохастического интеграла, управляемого двупараметрическим сильным мартингалом.
\end{abstract}

Ключевые слова и фразы: двупараметрический сильный мартингал, квадратическая вариация, стохастический интеграл.

1. Введение. Свойство измеримости по параметру квадратической вариации двупараметрического сильного мартингала и стохастического интеграла, управляемого таким мартингалом, имеет важное значение для построения и исследования стохастических интегральных уравнений [1]. Измеримость по параметру однопараметрических случайных процессов исследовалась многими авторами. Например, в работах [2] и [3] содержится ряд результатов о существовании измеримых по параметру модификаций случайных процессов, квадратических вариаций семимартингалов и управляемых ими стохастических интегралов. В двупараметрическом случае решение вопроса об измеримости по параметру имеет свои существенные особенности, не позволяющие воспользоваться известными результатами для однопараметрического случая. Некоторые результаты об измеримости по параметру стохастического интеграла, управляемого двупараметрическим сильным мартингалом, были анонсированы в [4].

Для обозначения элементов пространства $\mathbf{R}_{+}^{2}$ будем использовать только буквы $x$, $y, z, u, v$. Если в одном и том же выражении будут присутствовать $z, z_{1}$ и (или) $z_{2}$, то всегда будем считать, что $z_{1}$ - первый элемент, а $z_{2}$ - второй элемент вектора $z$ (аналогично для $x, y, u, v)$. Неравенства $x \leqslant y, x<y, x \geqslant y, x>y$ и структурные операции $x \vee y, x \wedge y$ полагаем определенными поэлементно; ] $x, y$ ] обозначает прямоугольник $] x, y]=\{u: x<u \leqslant y\}$ (пустой, если не выполнено условие $x<y$ ). Приращение функции $g: \mathbf{R}_{+}^{2} \rightarrow \mathbf{R}$ на непустом прямоугольнике $\left.] x, z\right]$ определено равенством $g(] x, z])=g(z)-g\left(x_{1}, z_{2}\right)-g\left(z_{1}, x_{2}\right)+g(x)$. Если $\left.] x, z\right]=\varnothing$, то $\left.\left.g(] x, z\right]\right)$ полагаем равным нулю. Если $\lim _{z \geqslant x \rightarrow z} g(x)=g(z)$, то функцию $g$ будем называть непрерывной слева. Если $\lim _{z \leqslant x \rightarrow z} g(x)=g(z)$, то функцию $g$ будем называть непрерывной справа.

Пусть $\mathbb{D}$ обозначает пространство всех таких функций $g: \mathbf{R}_{+}^{2} \rightarrow \mathbf{R}$, что: $g$ непрерывна справа; для каждого $z \in \mathbf{R}_{+}^{2} \backslash\left(\mathbf{R}_{+} \times\{0\}\right)$ существует предел $\lim _{x \rightarrow z, z_{1} \leqslant x_{1}, x_{2}<z_{2}} g(x)$; для каждого $z \in \mathbf{R}_{+}^{2} \backslash\left(\{0\} \times \mathbf{R}_{+}\right)$существует предел $\lim _{x \rightarrow z, x_{1}<z_{1}, z_{2} \leqslant x_{2}} g(x)$; для каждого $z>0$ существует предел $\lim _{z>x \rightarrow z} g(x)$. Для $g, g^{\prime}, g^{\prime \prime} \in \mathbb{D}$ определим

$$
\|g\|_{z}=\sup _{x \in[0, z]}|g(x)|, \quad \rho\left(g^{\prime}, g^{\prime \prime}\right)=\sum_{k=1}^{\infty} 2^{-k}\left(1 \wedge\left\|g^{\prime}-g^{\prime \prime}\right\|_{(k, k)}\right) .
$$

Пусть $\mathbb{D}_{0}$ обозначает пространство таких функций $g \in \mathbb{D}$, что $g\left(x_{1}, 0\right)=g\left(0, x_{2}\right)=0$ для любого $x$.

* Волгоградский государственный университет, математический факультет, пр-т Университетский, 100, 400062 Волгоград, Россия; e-mail: nkolodii@mail.ru 
Предположим, что $(\Theta, \mathscr{U})$ - произвольное измеримое пространство, $(\Omega, \mathscr{F}, \mathbf{P})-$ полное вероятностное пространство, $\mathbb{F}=\left(\mathscr{F}_{z}, z \in \mathbf{R}_{+}^{2}\right)-$ двупараметрическое семейство $\sigma$-алгебр, удовлетворяющее условиям:

1) если $x \leqslant z$, то $\mathscr{F}_{x} \subset \mathscr{F}_{z} \subset \mathscr{F}$;

2) $\mathscr{F}_{0}$ содержит все элементы $\mathscr{F}$ нулевой вероятности;

3) $\mathscr{F}_{z}=\bigcap_{x>z} \mathscr{F}_{x}$ для любого $z$;

4) для любых $x$ и $z \sigma$-алгебры $\mathscr{F}_{x}$ и $\mathscr{F}_{z}$ условно независимы относительно $\mathscr{F}_{x \wedge z}$.

В дальнейшем $\mathscr{T}$ и $\mathscr{P}$ обозначают соответственно $\sigma$-алгебры $\mathbb{F}$-прогрессивно измеримых и $\mathbb{F}$-предсказуемых подмножеств пространства $\mathbf{R}_{+}^{2} \times \Omega$ (см. [5]), а $\mathscr{M}_{S}^{2}=$ $\mathscr{M}_{S}^{2}(\mathbb{F}, \mathbf{P})$ обозначает пространство квадратично интегрируемых сильных $\mathbb{F}$-мартингалов, т.е. пространство таких $\mathscr{T}$-измеримых случайных полей $\left(\mu(z), z \in \mathbf{R}_{+}^{2}\right)$, что все траектории $\mu$ принадлежат $\mathbb{D}_{0}, \mathbf{E} \mu^{2}(z)<\infty$ и $\left.\left.\mathbf{E}\left\{\mu(] z, z^{\prime}\right]\right) \mid \mathscr{F}_{z}^{*}\right\}=0$ для всех $z \leqslant z^{\prime}$, где $\mathscr{F}_{z}^{*}=\sigma\left(\mathscr{F}_{x}, x_{1} \leqslant z_{1}\right.$ или $\left.x_{2} \leqslant z_{2}\right)$.

2. Об измеримости пределов последовательностей случайных полей. Приводимая ниже теорема 1 содержит результат о существовании измеримой по параметру модификации предела последовательности случайных полей с траекториями в $\mathbb{D}$. Близкие по смыслу утверждения для однопараметрических случайных процессов содержатся в работах [3] и [6], но без свойств регулярности их траекторий.

Теорема 1. Предположим, что выполнены следующие условия:

1) $\mathscr{C}-$ такая $\sigma$-алгебра подмножеств пространства $\Theta \times \mathbf{R}_{+}^{2} \times \Omega$, ито $\mathscr{C} \subset$ $\mathscr{U} \otimes \mathscr{B}\left(\mathbf{R}_{+}^{2}\right) \otimes \mathscr{F} u U \times \mathbf{R}_{+}^{2} \times \Omega \in \mathscr{C}$ для каждого $U \in \mathscr{U} ;$

2) $\left(\xi_{n}(\theta, z, \omega)\right)_{n \in \mathbf{N}}-$ такая последовательность $\mathscr{C} \mid \mathscr{B}(\mathbf{R})$-измеримьх функиий, ито $\xi_{n}(\theta, \cdot, \omega) \in \mathbb{D}$ для любих $\theta \in \Theta, \omega \in \Omega, n \in \mathbf{N} u$

$$
\lim _{r, j \rightarrow \infty} \mathbf{P}\left\{\left\|\xi_{r}(\theta, \cdot)-\xi_{j}(\theta, \cdot)\right\|_{z}>\varepsilon\right\}=0 \quad \text { для любых } \varepsilon>0, \theta \in \Theta, z \in \mathbf{R}_{+}^{2} .
$$

Тогда существует такая $\mathscr{C} \mid \mathscr{B}(\mathbf{R})$-измеримая функиия $\xi(\theta, z, \omega)$, ито для каждого $\theta \in \Theta$ траектории случайного поля $\xi(\theta, \cdot)$ п.н. принадлежат $\mathbb{D} u$

$$
\lim _{r \rightarrow \infty} \mathbf{P}\left\{\left\|\xi_{r}(\theta, \cdot)-\xi(\theta, \cdot)\right\|_{z}>\varepsilon\right\}=0 \quad \text { для любых } \varepsilon>0, \theta \in \Theta, z \in \mathbf{R}_{+}^{2} .
$$

Д о к а з а т е л ь с т в о. Заметим, что условие (1) эквивалентно условию

$$
\lim _{r, j \rightarrow \infty} \mathbf{P}\left\{\rho\left(\xi_{r}(\theta, \cdot), \xi_{j}(\theta, \cdot)\right)>\varepsilon\right\}=0 \quad \text { для любых } \varepsilon>0, \theta \in \Theta .
$$

Для $\varepsilon>0, \theta \in \Theta, n \in \mathbf{N}$ определим

$$
\varkappa_{n, \varepsilon}(\theta)=\sup _{r, j \geqslant n} \mathbf{P}\left\{\rho\left(\xi_{r}(\theta, \cdot), \xi_{j}(\theta, \cdot)\right)>\varepsilon\right\} .
$$

Так как $(\theta, \omega) \mapsto \rho\left(\xi_{r}(\theta, \cdot, \omega), \xi_{j}(\theta, \cdot, \omega)\right) \in(\mathscr{U} \otimes \mathscr{F}) \mid \mathscr{B}\left(\mathbf{R}_{+}\right)$, то

$$
\theta \mapsto \varkappa_{n, \varepsilon}(\theta) \in \mathscr{U} \mid \mathscr{B}([0,1]) .
$$

Для $\varepsilon>0, \delta>0, \theta \in \Theta$ определим

$$
n_{0}(\varepsilon, \delta, \theta)=\min \left\{n: \varkappa_{n, \varepsilon}(\theta) \leqslant \delta\right\} .
$$

Так как $\left\{n: \varkappa_{n, \varepsilon}(\theta) \leqslant \delta\right\} \neq \varnothing$ и $\left\{\theta: n_{0}(\varepsilon, \delta, \theta) \leqslant n\right\}=\left\{\theta: \varkappa_{n, \varepsilon}(\theta) \leqslant \delta\right\} \in \mathscr{U}$ для каждого $n \in \mathbf{N}$, то

$$
\theta \mapsto n_{0}(\varepsilon, \delta, \theta) \in \mathscr{U} \mid 2^{\mathbf{N}} .
$$

Пусть $n_{1}(\theta)=n_{0}(1 / 2,1, \theta), n_{k}(\theta)=\left(1+n_{k-1}(\theta)\right) \vee n_{0}\left(2^{-k}, k^{-2}, \theta\right)$. Определим последовательность случайных полей $\widehat{\xi}_{k}(\theta, z, \omega)=\xi_{n_{k}(\theta)}(\theta, z, \omega), k \in \mathbf{N}$. Тогда

$$
\begin{aligned}
& \left\{(\theta, z, \omega): \widehat{\xi}_{k}(\theta, z, \omega) \in B\right\} \\
& \quad=\bigcup_{n=1}^{\infty}\left\{(\theta, z, \omega): \xi_{n}(\theta, z, \omega) \in B\right\} \cap\left(\left\{\theta: n_{k}(\theta)=n\right\} \times \mathbf{R}_{+}^{2} \times \Omega\right) \in \mathscr{C}
\end{aligned}
$$


для любого $B \in \mathscr{B}(\mathbf{R})$. Кроме того, $\mathbf{P}\left\{\rho\left(\widehat{\xi}_{k+1}(\theta, \cdot), \widehat{\xi}_{k}(\theta, \cdot)\right)>2^{-k}\right\} \leqslant k^{-2}$ и $\widehat{\xi}_{k}(\theta, \cdot, \omega) \in$ $\mathbb{D}$ для любых $k \in \mathbf{N}, \theta \in \Theta, \omega \in \Omega$.

Пусть $\Omega_{\theta}=\liminf _{k \rightarrow \infty}\left\{\omega: \rho\left(\widehat{\xi}_{k+1}(\theta, \cdot, \omega), \widehat{\xi}_{k}(\theta, \cdot, \omega)\right) \leqslant 2^{-k}\right\}$. Тогда $\mathbf{P}\left(\Omega_{\theta}\right)=1$ для любого $\theta \in \Theta$. Для любых $\theta \in \Theta, \omega \in \Omega_{\theta}$ существует такой номер $k_{0}(\theta, \omega)$, что $\rho\left(\widehat{\xi}_{k+1}(\theta, \cdot, \omega), \widehat{\xi}_{k}(\theta, \cdot, \omega)\right) \leqslant 2^{-k}$ и поэтому $\sup _{m \in \mathbf{N}} \rho\left(\widehat{\xi}_{k+m}(\theta, \cdot, \omega), \widehat{\xi}_{k}(\theta, \cdot, \omega)\right) \leqslant 2^{-k+1}$ для всех $k \geqslant k_{0}(\theta, \omega)$. Следовательно, в каждой точке $(\theta, z, \omega) \in \Theta \times \mathbf{R}_{+}^{2} \times \Omega$ существует $\widehat{\xi}(\theta, z, \omega)=\lim _{k \rightarrow \infty} \widehat{\xi}_{k}(\theta, z, \omega) \mathrm{I}_{\Omega_{\theta}}(\omega)$. Согласно предыдущим построениям $\widehat{\xi}(\theta, \cdot, \omega) \in \mathbb{D}$ для любых $\theta \in \Theta$ и $\omega \in \Omega$. Кроме того, $\omega \mapsto \widehat{\xi}(\theta, z, \omega) \in \mathscr{F} \mid \mathscr{B}(\mathbf{R})$ для любых $\theta \in \Theta$, $z \in \mathbf{R}_{+}^{2}$. Следовательно,

$$
(z, \omega) \mapsto \widehat{\xi}(\theta, z, \omega) \in\left(\mathscr{F} \otimes \mathscr{B}\left(\mathbf{R}_{+}^{2}\right)\right) \mid \mathscr{B}(\mathbf{R}) \quad \text { для любого } \theta \in \Theta .
$$

Рассмотрим случайное поле

$$
\xi(\theta, z, \omega)=\lim _{k \rightarrow \infty} \widehat{\xi}_{k}(\theta, z, \omega) \mathrm{I}_{\left\{\exists \lim _{k \rightarrow \infty} \hat{\xi}_{k}\right\}}(\theta, z, \omega) .
$$

Очевидно, что $\xi \in \mathscr{C} \mid \mathscr{B}(\mathbf{R})$. Пусть $\widetilde{\Theta}=\left\{(\theta, z, \omega): \theta \in \Theta, \omega \in \Omega_{\theta}, z \in \mathbf{R}_{+}^{2}\right\}$. Так как $\widetilde{\Theta} \subset\left\{\exists \lim _{k \rightarrow \infty} \widehat{\xi}_{k}\right\}$, то $\xi(\theta, z, \omega)=\widehat{\xi}(\theta, z, \omega)$ для $(\theta, z, \omega) \in \widetilde{\Theta}$. Поэтому $\mathbf{P}\{\omega: \xi(\theta, \cdot, \omega)=$ $\widehat{\xi}(\theta, \cdot, \omega)\}=1$ и, значит, траектории случайного поля $(z, \omega) \mapsto \xi(\theta, z, \omega)$ п.н. принадлежат $\mathbb{D}$ для каждого $\theta \in \Theta$. Для любых $\theta \in \Theta, z \in \mathbf{R}_{+}^{2}, \varepsilon>0$

$$
\begin{aligned}
& \mathbf{P}\left\{\left\|\xi_{r}(\theta, \cdot)-\xi(\theta, \cdot)\right\|_{z}>\varepsilon\right\} \\
& \quad \leqslant \mathbf{P}\left\{\left\|\xi_{r}(\theta, \cdot)-\widehat{\xi}_{k}(\theta, \cdot)\right\|_{z}>\frac{\varepsilon}{2}\right\}+\mathbf{P}\left\{\left\|\widehat{\xi}_{k}(\theta, \cdot)-\widehat{\xi}(\theta, \cdot)\right\|_{z}>\frac{\varepsilon}{2}\right\}
\end{aligned}
$$

и поэтому $\lim _{r \rightarrow \infty} \mathbf{P}\left\{\left\|\xi_{r}(\theta, \cdot)-\xi(\theta, \cdot)\right\|_{z}>\varepsilon\right\}=0$. Теорема доказана.

Следствие 1. Если в условиях теоремь 1 предположить, что случайные поля $\xi_{n}(\theta, \cdot), n \in \mathbf{N}$, имеют непрерьвные траектории, то функииия $\xi$ может быть выбрана таким образом, ито траектории случайного поля $\xi(\theta, \cdot)$ будут п.н. непрерьвными для каждого $\theta \in \Theta$.

3. Измеримые по параметру модификации квадратических вариаций сильных мартингалов. Пусть $\mathscr{T}$ обозначает $\sigma$-алгебру таких подмножеств $C$ пространства $\Theta \times \mathbf{R}_{+}^{2} \times \Omega$, что $C \cap(\Theta \times[0, z] \times \Omega) \in \mathscr{U} \otimes \mathscr{B}([0, z]) \otimes \mathscr{F}_{z}$ для любых $z \in \mathbf{R}_{+}^{2}$. Заметим, что $\mathscr{U} \otimes \mathscr{T} \subset \widetilde{\mathscr{T}}$. Если $(\theta, \omega) \mapsto \nu(\theta, z, \omega) \in\left(\mathscr{U} \otimes \mathscr{F}_{z}\right) \mid \mathscr{B}(\mathbf{R})$ для каждого $z \in \mathbf{R}_{+}^{2}$ и $\nu(\theta, \cdot, \omega)$ непрерывна справа для любых $\theta$ и $\omega$, то $\nu \in \mathscr{T} \mid \mathscr{B}(\mathbf{R})$.

В дальнейшем $\mathscr{U}$-измеримым разбиением $\mathbf{R}_{+}^{2}$ будем называть такое семейство $\delta(\theta)$ прямоугольников $\left.\left.\delta_{i, j}(\theta)=\right] u_{i, j}(\theta), u_{i+1, j+1}(\theta)\right], i, j \in\{0,1, \ldots\}$, что

$$
\begin{gathered}
u_{0,0}=(0,0), \quad u_{i, j}(\theta)<u_{i+1, j+1}(\theta), \quad u_{i, j}(\theta)=\left(u_{i}^{\prime}(\theta), u_{j}^{\prime \prime}(\theta)\right), \\
\lim _{i \rightarrow \infty} u_{i}^{\prime}(\theta)=+\infty, \quad \lim _{j \rightarrow \infty} u_{j}^{\prime \prime}(\theta)=+\infty, \quad u_{i, j}(\cdot) \in \mathscr{U} \mid \mathscr{B}\left(\mathbf{R}_{+}^{2}\right) .
\end{gathered}
$$

Будем применять обозначение: $\left.\left.\delta_{i, j}^{x, y}(\theta)=\delta_{i, j}(\theta) \cap\right] x, y\right]$.

Теорема 2. Предположим, что $(\theta, x, \omega) \mapsto \mu(\theta, x, \omega) \in \widetilde{\mathscr{T}} \mid \mathscr{B}(\mathbf{R}) u \mu(\theta, \cdot) \in \mathscr{M}_{S}^{2}$ для каждого $\theta \in \Theta$. Тогда существует такая бункиия $(\theta, x, \omega) \mapsto \bar{\mu}(\theta, x, \omega) \in$ $\widetilde{\mathscr{T}} \mid \mathscr{B}\left(\mathbf{R}_{+}\right)$, ито для каждого фиксированного $\theta \in \Theta$ траектории случайного поля $\bar{\mu}(\theta, \cdot)$ п.н. принадлежат $\mathbb{D}_{0}$ и $\bar{\mu}(\theta, \cdot)$ является квадратической вариачией мартингала $\mu(\theta, \cdot)$, m.е. $\bar{\mu}(\theta, x)=\mathbf{P}-\lim _{|\delta| \rightarrow 0}[\mu(\theta)]_{x}^{\delta}$ для любьх $x \in \mathbf{R}_{+}^{2}, \theta \in \Theta$, где $[\mu(\theta)]_{x}^{\delta}=$ $\sum_{i, j}\left(\mu\left(\theta, \delta_{i, j}^{0, x}\right)\right)^{2}$.

Д о к а з а т е л ь с т в о. Для каждого фиксированного $\theta \in \Theta$, согласно теореме 1 из работы [7], сушествует такое поле $\left([\mu(\theta)]_{z}(\omega), z \in \mathbf{R}_{+}^{2}, \omega \in \Omega\right) \in \mathbb{F} \cap \mathbb{D}_{0}$, что $[\mu(\theta)]_{z}=\mathbf{P}-\lim _{|\delta| \rightarrow 0}[\mu(\theta)]_{z}^{\delta}$ для любого $z \in \mathbf{R}_{+}^{2}$. Пусть

$$
\begin{aligned}
\mathscr{D}_{n} & =\left\{\left(i \cdot 2^{-n}, j \cdot 2^{-n}\right), i, j=0,1, \ldots\right\}, \\
\delta_{n} & \left.\left.=\{]\left(i \cdot 2^{-n}, j \cdot 2^{-n}\right),\left((i+1) \cdot 2^{-n},(j+1) \cdot 2^{-n}\right)\right], i, j=0,1, \ldots\right\} .
\end{aligned}
$$


Заметим, что соотношения (1) и (2) из работы [7] для двоичных разбиений и в обозначениях настоящей статьи имеют вид

$$
\begin{array}{r}
\lim _{m \rightarrow \infty} \sup _{n>m} \mathbf{P}\left\{\sup _{x \in[0, z] \cap \mathscr{D}_{n}}\left|[\mu(\theta)]_{x}^{\delta_{n}}-[\mu(\theta)]_{x}^{\delta_{m}}\right|>\varepsilon\right\}=0, \\
\lim _{n \rightarrow \infty} \mathbf{P}\left\{\sup _{x \in[0, z] \cap \mathscr{D}_{n}}\left|[\mu(\theta)]_{x}-[\mu(\theta)]_{x}^{\delta_{n}}\right|>\varepsilon\right\}=0
\end{array}
$$

для любых $\varepsilon>0$ и $z \in \mathbf{R}_{+}^{2}$. Учитывая принадлежность траекторий полей $[\mu(\theta)]^{\delta_{n}}$ и $[\mu(\theta)]$. пространству $\mathbb{D}_{0}$, имеем сотношение

$$
\begin{aligned}
& \mathbf{P}\left\{\sup _{x \in[0, z]}\left|[\mu(\theta)]_{x}-[\mu(\theta)]_{x}^{\delta_{m}}\right|>\varepsilon\right\} \\
& \leqslant \sup _{n>m} \mathbf{P}\left\{\sup _{x \in[0, z] \cap \mathscr{D}_{n}}\left|[\mu(\theta)]_{x}-[\mu(\theta)]_{x}^{\delta_{n}}\right|>\frac{\varepsilon}{2}\right\} \\
& \quad+\sup _{n>m} \mathbf{P}\left\{\sup _{x \in[0, z] \cap \mathscr{D}_{n}}\left|[\mu(\theta)]_{x}^{\delta_{n}}-[\mu(\theta)]_{x}^{\delta_{m}}\right|>\frac{\varepsilon}{2}\right\} .
\end{aligned}
$$

Поэтому

$$
\lim _{m \rightarrow \infty} \mathbf{P}\left\{\sup _{x \in[0, z]}\left|[\mu(\theta)]_{x}-[\mu(\theta)]_{x}^{\delta_{m}}\right|>\varepsilon\right\}=0 \quad \text { для любых } \varepsilon>0, z \in \mathbf{R}_{+}^{2} .
$$

Следовательно,

$$
\lim _{r, j \rightarrow \infty} \mathbf{P}\left\{\rho\left([\mu(\theta)]^{\delta_{r}},[\mu(\theta)]^{\delta_{j}}\right)>\varepsilon\right\}=0 \quad \text { для любых } \varepsilon>0, \theta \in \Theta .
$$

Так как $(\theta, x, \omega) \mapsto[\mu(\theta)]_{x}^{\delta_{n}}(\omega) \in \widetilde{\mathscr{T}} \mid \mathscr{B}(\mathbf{R})$, то применение теоремы 1 с $\mathscr{C}=\widetilde{\mathscr{T}}$ завершает доказательство. Теорема доказана.

Следствие 2. Если выполнень условия теоремь 2 и $\Theta=\mathbf{R}_{+}^{2}, \mathscr{U}=\mathscr{B}\left(\mathbf{R}_{+}^{2}\right)$, то случайное поле $(x, \omega) \mapsto \bar{\mu}(x, x, \omega)$ является $\mathscr{T} \mid \mathscr{B}\left(\mathbf{R}_{+}\right)$-измеримым.

4. Некоторые пространства случайных полей. Настоящий пункт содержит утверждения, на которые опирается доказательство теоремы о существовании измеримой по параметру модификации стохастического интеграла от предсказуемого поля по квадратично интегрируемому сильному мартингалу. Основным утверждением является теорема 4, в которой содержится доказательство плотности пространства ступенчатых случайных полей над измеримыми по параметру $\theta$ разбиениями $\mathbf{R}_{+}^{2}$ в пространстве предсказуемых случайных полей, локально интегрируемых с квадратом по мере $\mathbf{P}(d \omega) \bar{\mu}(\theta, d x, \omega)$ для каждого $\theta \in \Theta$.

Теорема 3. Пусть $\mathbb{B}-$ класс функций $\beta: \Theta \times \mathbf{R}_{+}^{2} \times \Omega \rightarrow \mathbf{R}$, удовлетворяюших условиям:

$(\mathbb{B} 1) \sup _{z, \omega}|\beta(\theta, z, \omega)|<\infty$ для любих $\beta \in \mathbb{B}, \theta \in \Theta$

$(\mathbb{B} 2)$ если $\beta^{\prime}, \beta^{\prime \prime} \in \mathbb{B}$ и $a^{\prime}, a^{\prime \prime} \in \mathscr{U} \mid \mathscr{B}(\mathbf{R})$, mo $a^{\prime} \beta^{\prime}+a^{\prime \prime} \beta^{\prime \prime} \in \mathbb{B} ;$

$(\mathbb{B} 3) \mathbb{B}$ содержит все функиии $\beta$ со следуюшими свойствами:

1) $\beta(\theta, z, \cdot) \in \mathscr{F}_{z} \mid \mathscr{B}(\mathbf{R})$ для любих $\theta$ u $z$,

2) $z \mapsto \beta(\theta, z, \omega)$ непрерывна слева для любых $\theta$ u $\omega$;

$(\mathbb{B} 4)$ если $\left(\beta_{n}\right)_{n \in \mathbf{N}} \subset \mathbb{B} u \beta_{n}(\theta, z, \omega) \leqslant \beta_{n+1}(\theta, z, \omega), \sup _{n, z, \omega}\left|\beta_{n}(\theta, z, \omega)\right|<\infty \partial л я$ каждого $\theta \in \Theta$, то $\lim _{n \rightarrow \infty} \beta_{n} \in \mathbb{B}$.

Тогда $\mathbb{B}$ содержит все $(\mathscr{U} \otimes \mathscr{P}) \mid \mathscr{B}(\mathbf{R})$-измеримье и для каждого $\theta \in \Theta$ ограниченные поля $(\theta,(z, \omega)) \mapsto \beta(\theta, z, \omega)$.

В монографии [8] содержится аналогичное теореме 3 предложение 5.1 для случайных процессов, определенных на $\mathbf{R}_{+}$, без условий и утверждений измеримой зависимости от параметра. Доказательство теоремы 3 в общих чертах похоже на доказательство этого предложения и потому здесь не приводится. 
Лемма 1. $\mathscr{U} \otimes \mathscr{P}$ совпадает с минимальной $\sigma$-алгеброй $\widetilde{P}$, относительно которой измеримь все случайнье поля $\beta: \Theta \times \mathbf{R}_{+}^{2} \times \Omega \rightarrow \mathbf{R}$, удовлетворяюшие условиям:

$1^{\circ} .(\theta, \omega) \mapsto \beta(\theta, z, \omega) \in\left(\mathscr{U} \otimes \mathscr{F}_{z}\right) \mid \mathscr{B}(\mathbf{R})$ для каждого $z \in \mathbf{R}_{+}^{2} ;$

$2^{\circ} . z \mapsto \beta(\theta, z, \omega)$ непрерывна слева для любих $\theta$ и $\omega$.

Д о к а з а т е л ь с т в о. Предположим, что $\beta$ удовлетворяет условиям $1^{\circ}$ и $2^{\circ}$, и рассмотрим последовательность случайных полей

$$
\begin{aligned}
\widehat{\beta}_{n}(\theta, z, \omega)= & \sum_{i, j=0}^{\infty} \beta\left(\theta,\left(i \cdot 2^{-n}, j \cdot 2^{-n}\right), \omega\right) \mathrm{I}_{]\left(i \cdot 2^{-n}, j \cdot 2^{-n}\right) ;\left((i+1) \cdot 2^{-n},(j+1) \cdot 2^{-n}\right)\right]}(z) \\
& +\beta(\theta, 0, \omega) \mathrm{I}_{\{(0,0)\}}(z)+\sum_{i=0}^{\infty} \beta\left(\theta,\left(i \cdot 2^{-n}, 0\right), \omega\right) \mathrm{I}_{] i \cdot 2^{-n},(i+1) \cdot 2^{-n}\right] \times\{0\}}(z) \\
& +\sum_{j=0}^{\infty} \beta\left(\theta,\left(0, j \cdot 2^{-n}\right), \omega\right) \mathrm{I}_{\left.\{0\} \times] j \cdot 2^{-n},(j+1) \cdot 2^{-n}\right]}(z), \quad n \in \mathbf{N} .
\end{aligned}
$$

Из условия $1^{\circ}$ следует, что для любых $u, v \in \mathbf{R}_{+}^{2}$ и $s, t \in \mathbf{R}_{+}$случайные поля $(\theta,(z, \omega)) \mapsto \beta(\theta, u, \omega) \mathrm{I}_{] u, v]}(z),(\theta,(z, \omega)) \mapsto \beta(\theta,(s, 0), \omega) \mathrm{I}_{] s, t] \times\{0\}}(z)$ и $(\theta,(z, \omega)) \mapsto$ $\beta(\theta,(0, s), \omega) \mathrm{I}_{\{0\} \times] s, t]}^{(z)}$ являются $(\mathscr{U} \otimes \mathscr{P}) \mid \mathscr{B}(\mathbf{R})$-измеримыми, поэтому $(\theta,(z, \omega)) \mapsto$ $\widehat{\beta}_{n}(\theta, z, \omega) \in(\mathscr{U} \otimes \mathscr{P}) \mid \mathscr{B}(\mathbf{R})$. Так как $\lim _{n \rightarrow \infty} \widehat{\beta}_{n}(\theta, z, \omega)=\beta(\theta, z, \omega)$ в каждой точке $(\theta, z, \omega)$, то $(\theta,(z, \omega)) \mapsto \beta(\theta, z, \omega) \in(\mathscr{U} \otimes \mathscr{P}) \mid \mathscr{B}(\mathbf{R})$. Следовательно, $\widetilde{P} \subset \mathscr{U} \otimes \mathscr{P}$.

Отметим, что $\mathscr{P}$ совпадает с минимальной $\sigma$-алгеброй, порожденной классом множеств

$$
\begin{aligned}
\mathscr{P}_{0}= & \left.\{] x, z] \times A: x, z \in \mathbf{R}_{+}^{2}, x<z, A \in \mathscr{F}_{x}\right\} \\
& \left.\cup\{\{0\} \times] s, t] \times A: s, t \in \mathbf{R}_{+}, s<t, A \in \mathscr{F}_{(0, s)}\right\} \\
& \left.\cup\{] s, t] \times\{0\} \times A: s, t \in \mathbf{R}_{+}, s<t, A \in \mathscr{F}_{(s, 0)}\right\} \\
& \cup\left\{\{0\} \times A: A \in \mathscr{F}_{0}\right\} .
\end{aligned}
$$

Если $U \in \mathscr{U}$ и $B \in \mathscr{P}_{0}$, то случайное поле $\beta(\theta, z, \omega)=\mathrm{I}_{U \times B}(\theta, z, \omega)$ удовлетворяет условиям $1^{\circ}$ и $2^{\circ}$ и, значит, $U \times B \in \widetilde{\mathscr{P}}$. Следовательно, $\mathscr{U} \times \mathscr{P}_{0} \subset \widetilde{\mathscr{P}}$ и поэтому $\mathscr{U} \otimes \mathscr{P} \subset \widetilde{\mathscr{P}}$. Лемма доказана.

Лемма 2. Пусть $(\theta,(z, \omega)) \mapsto \beta(\theta, z, \omega) \in(\mathscr{U} \otimes \mathscr{P}) \mid \mathscr{B}(\mathscr{R})$, функиии $u(\cdot)$, $v(\cdot)$ являются $\mathscr{U} \mid \mathscr{B}\left(\mathbf{R}_{+}^{2}\right)$-измеримыми и функции $s(\cdot), t(\cdot)$ являются $\mathscr{U} \mid \mathscr{B}\left(\mathbf{R}_{+}\right)$измеримьми. Тогда случайные поля

$$
\begin{aligned}
& (\theta,(z, \omega)) \mapsto \beta(\theta, u(\theta), \omega) \mathrm{I}_{] u(\theta), v(\theta)]}(z), \\
& (\theta,(z, \omega)) \mapsto \beta(\theta,(s(\theta), 0), \omega) \mathrm{I}_{] s(\theta), t(\theta)] \times\{0\}}(z), \\
& (\theta,(z, \omega)) \mapsto \beta(\theta,(0, s(\theta)), \omega) \mathrm{I}_{\{0\} \times] s(\theta), t(\theta)]}(z)
\end{aligned}
$$

являются $(\mathscr{U} \otimes \mathscr{P}) \mid \mathscr{B}(\mathbf{R})$-измеримьли.

Д о к а з а т е ль с т в о. Так как $\mathscr{U} \otimes \mathscr{P} \subset \widetilde{\mathscr{T}}$, то

$$
\{(\theta, u, v, \omega): u<z \leqslant v, \beta(\theta, u, \omega) \in B\} \in \mathscr{U} \otimes \mathscr{B}([0, z]) \otimes \mathscr{B}\left(\mathbf{R}_{+}^{2}\right) \otimes \mathscr{F}_{z}
$$

для любых $z \in \mathbf{R}_{+}^{2}, B \in \mathscr{B}(\mathbf{R})$. Поэтому для каждого $z \in \mathbf{R}_{+}^{2}$

$$
(\theta, u, v, \omega) \mapsto \beta(\theta, u, \omega) \mathrm{I}_{] u, v]}(z) \in\left(\mathscr{U} \otimes \mathscr{B}\left(\mathbf{R}_{+}^{2}\right) \otimes \mathscr{B}\left(\mathbf{R}_{+}^{2}\right) \otimes \mathscr{F}_{z}\right) \mid \mathscr{B}(\mathbf{R}) .
$$

Так как функция $z \mapsto \beta(\theta, u, \omega) \mathrm{I}_{] u, v]}(z)$ непрерывна слева для любых $\theta, u, v, \omega$, то в силу леммы 1 , с заменой $(\Theta, \mathscr{U})$ на $\left(\Theta \times \mathbf{R}_{+}^{2} \times \mathbf{R}_{+}^{2}, \mathscr{U} \otimes \mathscr{B}\left(\mathbf{R}_{+}^{2}\right) \otimes \mathscr{B}\left(\mathbf{R}_{+}^{2}\right)\right)$, справедливо соотношение

$$
(\theta, u, v,(z, \omega)) \mapsto \beta(\theta, u, \omega) \mathrm{I}_{] u, v]}(z) \in\left(\mathscr{U} \otimes \mathscr{B}\left(\mathbf{R}_{+}^{2}\right) \otimes \mathscr{B}\left(\mathbf{R}_{+}^{2}\right) \otimes \mathscr{P}\right) \mid \mathscr{B}(\mathbf{R}) .
$$


Так как $u(\cdot), v(\cdot) \in \mathscr{U} \mid \mathscr{B}\left(\mathbf{R}_{+}^{2}\right)$, то из (2) очевидным образом следует $(\mathscr{U} \otimes \mathscr{P}) \mid \mathscr{B}(\mathbf{R})$ измеримость отображения $(\theta,(z, \omega)) \mapsto \beta(\theta, u(\theta), \omega) \mathrm{I}_{] u(\theta), v(\theta)]}(z)$. Доказательство остальных утверждений данной леммы аналогично. Лемма доказана.

Пусть $\mathbb{B}_{0}$ обозначает пространство всех случайных полей вида

$$
\begin{aligned}
\widehat{\beta}(\theta, z, \omega)= & \gamma^{\delta(\theta)}(\theta, z, \omega) \\
= & \gamma(\theta,(0,0), \omega) \mathrm{I}_{\{(0,0)\}}(z)+\sum_{i=0}^{\infty} \gamma\left(\theta,\left(u_{i}^{\prime}(\theta), 0\right), \omega\right) \mathrm{I}_{] u_{i}^{\prime}(\theta), u_{i+1}^{\prime}(\theta)\right] \times\{0\}}(z) \\
& +\sum_{j=0}^{\infty} \gamma\left(\theta,\left(0, u_{j}^{\prime \prime}(\theta)\right), \omega\right) \mathrm{I}_{\left.\{0\} \times] u_{j}^{\prime \prime}(\theta), u_{j+1}^{\prime \prime}(\theta)\right]}(z) \\
& +\sum_{i, j=0}^{\infty} \gamma\left(\theta, u_{i, j}(\theta), \omega\right) \mathrm{I}_{] u_{i, j}(\theta), u_{i+1, j+1}(\theta)\right]}(z),
\end{aligned}
$$

где $(\theta,(z, \omega)) \mapsto \gamma(\theta, z, \omega) \in(\mathscr{U} \otimes \mathscr{P}) \mid \mathscr{B}(\mathbf{R})$ и $\delta(\theta)$ есть $\mathscr{U}$-измеримое разбиение $\mathbf{R}_{+}^{2}$.

В дальнейшем элемент пространства $\mathbb{B}_{0}$ будет обозначаться $\widehat{\beta}$, либо $\gamma^{\delta}$ в зависимости от удобства и необходимости указывать разбиение, участвующее в определении этого элемента. Заметим, что в силу леммы 2 случайное поле $\widehat{\beta}(\theta, z, \omega)$ является $(\mathscr{U} \otimes \mathscr{P}) \mid \mathscr{B}(\mathbf{R})$-измеримым. Если $\widehat{\beta}_{1}$ и $\widehat{\beta}_{2}$ - два элемента пространства $\mathbb{B}_{0}$, то, не умаляя общности, можно считать, что $\widehat{\beta}_{1}$ и $\widehat{\beta}_{2}$ определены над одним и тем же разбиением $\delta(\cdot)$.

Если $\mu \in \mathscr{M}_{S}^{2}$, то $\mathbb{B}_{2}(\mu)$ обозначает пространство всех таких случайных полей $(z, \omega) \mapsto \beta(z, \omega) \in \mathscr{P} \mid \mathscr{B}(\mathbf{R})$, что $|\beta|_{z}=\left(\mathbf{E} \int_{0, z]} \beta^{2}(x) \bar{\mu}(d x)\right)^{1 / 2}<\infty$ для каждого $z \in \mathbf{R}_{+}^{2}$. Для $\beta^{\prime}, \beta^{\prime \prime} \in \mathbb{B}_{2}(\mu)$ пусть $\varrho_{\mu}\left(\beta^{\prime}, \beta^{\prime \prime}\right)=\sum_{k=1}^{\infty} 2^{-k}\left[1 \wedge\left|\beta^{\prime}-\beta^{\prime \prime}\right|_{(k, k)}\right]$.

Теорема 4. Предположим, ито $(\theta, x, \omega) \mapsto \mu(\theta, x, \omega) \in \widetilde{\mathscr{T}} \mid \mathscr{B}(\mathbf{R}), \mu(\theta, \cdot) \in \mathscr{M}_{S}^{2}$, $(\theta,(x, \omega)) \mapsto \beta(\theta, x, \omega) \in(\mathscr{U} \otimes \mathscr{P}) \mid \mathscr{B}(\mathbf{R})$ u $\beta(\theta, \cdot) \in \mathbb{B}_{2}(\mu(\theta, \cdot))$ для каждого $\theta \in \Theta$.

Тогда существует такая последовательность случайньх полей $\left(\widehat{\beta}_{n}\right)_{n \in \mathbf{N}} \subset \mathbb{B}_{0}$, ито $\lim _{n \rightarrow \infty} \varrho_{\mu(\theta, \cdot)}\left(\widehat{\beta}_{n}(\theta, \cdot), \beta(\theta, \cdot)\right)=0$ для каждого $\theta \in \Theta$.

Д о к а з а т е л ь с т в о. Рассмотрим класс $\mathbb{B}$ таких полей $\beta \in(\mathscr{U} \otimes \mathscr{P}) \mid \mathscr{B}(\mathbf{R})$, что $\sup _{z, \omega}|\beta(\theta, z, \omega)|<\infty$ и существует такая последовательность случайных полей $\left(\widehat{\beta}_{n}\right)_{n \in \mathbf{N}} \subset \mathbb{B}_{0}$, что $\lim _{n \rightarrow \infty} \rho_{\mu(\theta, \cdot)}\left(\widehat{\beta}_{n}(\theta, \cdot), \beta(\theta, \cdot)\right)=0$ для каждого $\theta \in \Theta$. Отметим, что если $\beta \in \mathbb{B}$, то $\beta(\theta, \cdot) \in \mathbb{B}_{2}(\mu(\theta, \cdot))$ для каждого $\theta \in \Theta$.

Класс $\mathbb{B}$ удовлетворяет условиям $(\mathbb{B} 1)-(\mathbb{B} 3)$ теоремы 3 . Покажем, что $\mathbb{B}$ удовлетворяет условию $(\mathbb{B} 4)$.

Предположим, что $\beta_{n} \in \mathbb{B}, \beta_{n}(\theta, z, \omega) \leqslant \beta_{n+1}(\theta, z, \omega), \bar{\beta}_{\theta}=\sup _{n, z, \omega}\left|\beta_{n}(\theta, z, \omega)\right|<$ $\infty$, и пусть $\beta=\lim _{n \rightarrow \infty} \beta_{n}$. Тогда: $\sup _{z, \omega}|\beta(\theta, z, \omega)| \leqslant \bar{\beta}_{\theta}<\infty ;|\beta(\theta, \cdot)|_{z} \leqslant$ $(\mathbf{E} \bar{\mu}(\theta, z))^{1 / 2} \bar{\beta}_{\theta}<\infty$ и поэтому $\beta \in \mathbb{B}_{2} ; \lim _{n \rightarrow \infty}\left|\beta_{n}(\theta, \cdot)-\beta(\theta, \cdot)\right|_{z}=0$ по теореме Лебега о мажорируемой сходимости и поэтому $\lim _{n \rightarrow \infty} \varrho_{\mu(\theta, \cdot)}\left(\beta_{n}(\theta, \cdot), \beta(\theta, \cdot)\right)=$ 0 . Кроме того, существует такое семейство полей $\left(\widehat{b}_{n, r}\right)_{n, r \in \mathbf{N}} \subset \mathbb{B}_{0}$, что $\lim _{r \rightarrow \infty} \varrho_{\mu(\theta, \cdot)}\left(\widehat{b}_{n, r}(\theta, \cdot), \beta_{n}(\theta, \cdot)\right)=0$ для каждого $n \in \mathbf{N}$ и каждого $\theta \in \Theta$.

Определим $n_{k}(\theta)=\min \left\{n: \varrho_{\mu(\theta, \cdot)}\left(\beta_{n}(\theta, \cdot), \beta(\theta, \cdot)\right) \leqslant 1 / k\right\}, k \in \mathbf{N}, \theta \in \Theta$. Так как $\left\{n: \varrho_{\mu(\theta, \cdot)}\left(\beta_{n}(\theta, \cdot), \beta(\theta, \cdot)\right) \leqslant 1 / k\right\} \neq \varnothing$ и

$$
\left\{\theta: n_{k}(\theta) \leqslant n\right\}=\left\{\theta: \varrho_{\mu(\theta, \cdot)}\left(\beta_{n}(\theta, \cdot), \beta(\theta, \cdot)\right) \leqslant \frac{1}{k}\right\} \in \mathscr{U},
$$

то $n_{k}(\cdot) \in \mathscr{U} \mid 2^{\mathbf{N}}$. Поэтому для любого $B \in \mathscr{B}\left(\mathbf{R}_{+}^{2}\right)$

$$
\begin{aligned}
& \left\{(\theta,(z, \omega)): \beta_{n_{k}(\theta)}(\theta, z, \omega) \in B\right\} \\
& \quad=\bigcup_{n=1}^{\infty}\left\{(\theta,(z, \omega)): \beta_{n}(\theta, z, \omega) \in B\right\} \cap\left(\left\{\theta: n_{k}(\theta)=n\right\} \times\left(\mathbf{R}_{+}^{2} \times \Omega\right)\right) \in \mathscr{U} \otimes \mathscr{P} .
\end{aligned}
$$


Таким образом, $(\theta,(z, \omega)) \mapsto \beta_{n_{k}(\theta)}(\theta, z, \omega) \in(\mathscr{U} \otimes \mathscr{P}) \mid \mathscr{B}(\mathbf{R}) . \quad$ Кроме того, $\left(\widehat{b}_{n_{k}, r}\right)_{r \in \mathbf{N}} \subset \mathbb{B}_{0}$ и $\lim _{r \rightarrow \infty} \varrho_{\mu(\theta, \cdot)}\left(\widehat{b}_{n_{k}(\theta), r}(\theta, \cdot), \beta_{n_{k}(\theta)}(\theta, \cdot)\right)=0$ для каждого $\theta \in \Theta$.

Теперь определим $r_{k}(\theta)=\min \left\{r: \varrho_{\mu(\theta, \cdot)}\left(\widehat{b}_{n_{k}(\theta), r}(\theta, \cdot), \beta_{n_{k}(\theta)}(\theta, \cdot)\right) \leqslant 1 / k\right\}$. Тогда $r_{k}(\cdot) \in \mathscr{U} \mid 2^{\mathbf{N}}$ и $\widetilde{\beta}_{k}(\theta, z, \omega)=\widehat{b}_{n_{k}(\theta), r_{k}(\theta)}(\theta, z, \omega)$ является элементом пространства $\mathbb{B}_{0}$ для каждого $k \in \mathbf{N}$. Очевидно, что $\lim _{k \rightarrow \infty} \varrho_{\mu(\theta, \cdot)}\left(\widetilde{\beta}_{k}(\theta, \cdot), \beta(\theta, \cdot)\right)=0$. Следовательно, $\beta \in \mathbb{B}$ и, значит, условие ( $\mathbb{B} 4)$ выполнено.

Согласно теореме 3 класс $\mathbb{B}$ содержит все $(\mathscr{U} \otimes \mathscr{P}) \mid \mathscr{B}(\mathbf{R})$-измеримые и для каждого $\theta \in \Theta$ ограниченные поля $\beta(\theta, z, \omega)$.

Предположим, что для каждого $\theta \in \Theta$ случайное поле $\beta(\theta, \cdot)$ является элементом пространства $\mathbb{B}_{2}(\mu(\theta, \cdot))$. Для $m \in \mathbf{N}$ определим $\beta_{m}(\theta, z, \omega)=(\beta(\theta, z, \omega) \vee(-m)) \wedge m$. Согласно предыдущей части доказательства теоремы, существует такое семейство полей $\left(\widehat{\beta}_{m, r}\right)_{m, r \in \mathbf{N}} \subset \mathbb{B}_{0}$, что $\lim _{r \rightarrow \infty} \rho_{\mu(\theta, \cdot)}\left(\widehat{\beta}_{m, r}(\theta, \cdot), \beta_{m}(\theta, \cdot)\right)=0$ для каждого $m \in \mathbf{N}$ и каждого $\theta \in \Theta$.

Определим $m_{k}(\theta)=\min \left\{m: \rho_{\mu(\theta, \cdot)}\left(\beta_{m}(\theta, \cdot), \beta(\theta, \cdot)\right) \leqslant 1 / k\right\}$. Тогда $m_{k}(\cdot) \in \mathscr{U} \mid 2^{\mathbf{N}}$, $(\theta,(z, \omega)) \mapsto \beta_{m_{k}(\theta)}(\theta, z, \omega) \in(\mathscr{U} \otimes \mathscr{P}) \mid \mathscr{B}(\mathbf{R}),\left(\widehat{\beta}_{m_{k}, r}\right)_{r \in \mathbf{N}} \subset \mathbb{B}_{0}$ и

$$
\lim _{r \rightarrow \infty} \rho_{\mu(\theta, \cdot)}\left(\widehat{\beta}_{m_{k}(\theta), r}(\theta, \cdot), \beta_{m_{k}(\theta)}(\theta, \cdot)\right)=0
$$

Пусть $r_{k}(\theta)=\min \left\{r: \rho_{\mu(\theta, \cdot)}\left(\beta_{m_{k}(\theta), r}(\theta, \cdot), \widehat{\beta}_{m_{k}(\theta)}(\theta, \cdot)\right) \leqslant 1 / k\right\}$. Ясно, что $r_{k}(\cdot) \in \mathscr{U} \mid 2^{\mathbf{N}}$, и поэтому $\widetilde{\beta}_{k}(\theta, z, \omega)=\widehat{\beta}_{m_{k}}(\theta), r_{k}(\theta)(\theta, z, \omega)$ является элементом пространства $\mathbb{B}_{0}$ для каждого $k \in \mathbf{N}$. Теперь очевидно, что $\lim _{k \rightarrow \infty} \rho_{\mu(\theta, \cdot)}\left(\widetilde{\beta}_{k}(\theta, \cdot), \beta(\theta, \cdot)\right)=0$. Теорема доказана.

\section{5. Измеримые по параметру модификации стохастических интегра-} лов.

Теорема 5. Предположим, что выполнены следующие условия:

$$
\begin{gathered}
(\theta,(x, \omega)) \mapsto \beta(\theta, x, \omega) \in \mathscr{U} \otimes \mathscr{P}|\mathscr{B}(\mathbf{R}) ; \quad(\theta, x, \omega) \mapsto \mu(\theta, x, \omega) \in \widetilde{\mathscr{T}}| \mathscr{B}(\mathbf{R}) ; \\
\mu(\theta, \cdot) \in \mathscr{M}_{S}^{2} \quad \text { для каждого } \theta \in \Theta ; \\
\mathbf{E} \int_{] 0, x]} \beta^{2}(\theta, u) \bar{\mu}(\theta, d u)<\infty \quad \text { для любьх } \theta \in \Theta, x \in \mathbf{R}_{+}^{2} .
\end{gathered}
$$

Тогда существует такая функиия $\Psi(\theta, x, \omega): \Theta \times \mathbf{R}_{+}^{2} \times \Omega \mapsto \mathbf{R}$, ито:

1) $(\theta, x, \omega) \mapsto \Psi(\theta, x, \omega) \in \widetilde{\mathscr{T}} \mid \mathscr{B}(\mathbf{R})$;

2) $\Psi(\theta, x)$ является модификачией стохастического интеграла на прямоугольнике $] 0, x]$ от предсказуемого поля $\beta(\theta, \cdot)$ по квадратично интегрируемому сильному мартингалу $\mu(\theta, \cdot)$ :

$$
\Psi(\theta, x)=\int_{] 0, x]} \beta(\theta, u) \mu(\theta, d u) \quad \text { п.н. для любих } x \in \mathbf{R}_{+}^{2}, \theta \in \Theta
$$

3) для каждого фиксированного $\theta \in \Theta$ случайное поле $\Psi(\theta, \cdot)$ является элементом класса $\mathscr{M}_{S}^{2}$, и квадратическая вариачия $\Psi(\theta, \cdot)$ на прямоугольнике $\left.] 0, x\right]$ равна $\int_{] 0, x]} \beta^{2}(\theta, u) \bar{\mu}(\theta, d u)$.

Д о к а з а т е л ь с т в о. Для произвольного элемента $\gamma^{\delta}$ пространства случайных полей $\mathbb{B}_{0}$ определим

$$
\widetilde{\Psi}\left(\gamma^{\delta}, \theta, z, \omega\right)=\int_{0}^{z} \gamma^{\delta}(\theta, x) \mu(\theta, d x)=\sum_{i, j: u_{i, j}<z} \gamma\left(\theta, u_{i, j}(\theta), \omega\right) \mu\left(\theta, \delta_{i, j}^{0, z}(\theta), \omega\right) .
$$

Заметим, что $\gamma\left(\theta, u_{i, j}(\theta), \omega\right) \mu\left(\theta, \delta_{i, j}^{0, z}(\theta), \omega\right)=\gamma\left(\theta, u_{i, j}(\theta) \wedge z, \omega\right) \mu\left(\theta, \delta_{i, j}^{0, z}(\theta), \omega\right)$. Так как для каждого $z \in \mathbf{R}_{+}^{2}$

$$
\begin{aligned}
(\theta, u, v, \omega) & \mapsto \mu(\theta,] u, v] \cap] 0, z], \omega) \in\left(\mathscr{U} \otimes \mathscr{B}\left(\mathbf{R}_{+}^{2}\right) \otimes \mathscr{B}\left(\mathbf{R}_{+}^{2}\right) \otimes \mathscr{F}_{z}\right) \mid \mathscr{B}(\mathbf{R}), \\
(\theta, u, \omega) & \mapsto \gamma(\theta, u \wedge z, \omega) \in\left(\mathscr{U} \otimes \mathscr{B}\left(\mathbf{R}_{+}^{2}\right) \otimes \mathscr{F}_{z}\right) \mid \mathscr{B}(\mathbf{R}),
\end{aligned}
$$


то отображения $(\theta, \omega) \mapsto \mu\left(\theta, \delta_{i, j}^{0, z}(\theta), \omega\right)$ и $(\theta, \omega) \mapsto \gamma\left(\theta, u_{i, j}(\theta) \wedge z, \omega\right)$ являются $(\mathscr{U} \otimes$ $\left.\mathscr{F}_{z}\right) \mid \mathscr{B}(\mathbf{R})$-измеримыми. Следовательно,

$$
(\theta, \omega) \mapsto \gamma\left(\theta, u_{i, j}(\theta), \omega\right) \mu\left(\theta, \delta_{i, j}^{0, z}(\theta), \omega\right) \in\left(\mathscr{U} \otimes \mathscr{F}_{z}\right) \mid \mathscr{B}(\mathbf{R})
$$

Учитывая равенство $\lim _{z \rightarrow u_{i, j}(\theta) \leqslant z} \mu\left(\theta, \delta_{i, j}^{0, z}(\theta), \omega\right)=0$, замечаем, что отображение $z \mapsto$ $\widetilde{\Psi}\left(\gamma^{\delta}, \theta, z, \omega\right)$ непрерывно справа для любых $\theta \in \Theta$ и $\omega \in \Omega$. Следовательно, $(\theta, z, \omega) \mapsto$ $\widetilde{\Psi}\left(\gamma^{\delta}, \theta, z, \omega\right) \in \widetilde{\mathscr{T}} \mid \mathscr{B}(\mathbf{R})$.

Заметим теперь, что $\widetilde{\Psi}\left(\gamma^{\delta}, \theta, \cdot, \omega\right) \in \mathbb{D}, \widetilde{\Psi}\left(\gamma^{\delta}, \theta, \cdot\right) \in \mathscr{M}_{S}^{2}$ и квадратическая вариация $\widetilde{\Psi}\left(\gamma^{\delta}, \theta, \cdot\right)$ равна

$$
\left[\widetilde{\Psi}\left(\gamma^{\delta}, \theta, \cdot\right)\right]_{z}=\sum_{i, j: u_{i, j}<z}\left(\gamma\left(\theta, u_{i, j}(\theta)\right)\right)^{2} \bar{\mu}\left(\theta, \delta_{i, j}^{0, z}(\theta)\right) .
$$

Согласно неравенству Каироли (см. [9]),

$$
\mathbf{E}\left\|\widetilde{\Psi}\left(\gamma^{\delta}, \theta, \cdot\right)\right\|_{z}^{2} \leqslant 16\left|\gamma^{\delta(\theta)}(\theta, \cdot)\right|_{z}^{2} \quad \text { для любых } \theta \in \Theta, z \in \mathbf{R}_{+}^{2} .
$$

Из теоремы 4 вытекает, что существует такая последовательность случайных полей $\left(\gamma_{n}^{\delta_{n}}\right)_{n \in \mathbf{N}} \subset \mathbb{B}_{0}$, что $\lim _{n \rightarrow \infty} \rho_{\mu(\theta, \cdot)}\left(\gamma_{n}^{\delta_{n}(\theta)}(\theta, \cdot), \beta(\theta, \cdot)\right)=0$ для каждого $\theta \in \Theta$, поэтому из (3) следует, что

$$
\lim _{r, j \rightarrow \infty} \mathbf{E}\left\|\widetilde{\Psi}\left(\gamma_{r}^{\delta_{r}}, \theta, \cdot\right)-\widetilde{\Psi}\left(\gamma_{j}^{\delta_{j}}, \theta, \cdot\right)\right\|_{z}^{2}=0 \quad \text { для любых } \theta \in \Theta, z \in \mathbf{R}_{+}^{2} .
$$

Согласно теореме 1 существует такое $\widetilde{\mathscr{T}} \mid \mathscr{B}(\mathbf{R})$-измеримое поле $\Psi(\theta, z, \omega)$, что траектории $\Psi(\theta, \cdot)$ п.н. принадлежат $\mathbb{D}$ и

$$
\lim _{r} \mathbf{E}\left\|\widetilde{\Psi}\left(\gamma_{r}^{\delta_{r}}, \theta, \cdot\right)-\Psi(\theta, \cdot)\right\|_{z}^{2}=0 \quad \text { для любых } \theta \in \Theta, z \in \mathbf{R}_{+}^{2} .
$$

Очевидно, что поле $\Psi$ удовлетворяет утверждениям 2) и 3 ) данной теоремы. Теорема доказана.

Следствие 3. Если в условиях теоремь 5 предположить, что для каждого $\theta \in$ $\Theta$ траектории сильного мартингала $\mu(\theta, \cdot)$ п.н. непрерывнь, то функиия $\Psi$ может быть выбрана такой, что траектории $\Psi(\theta, \cdot)$ также п.н. непрерывны.

Следствие 4. Если выполнень условия теоремь 5 и $\Theta=\mathbf{R}_{+}^{2}, \mathscr{U}=\mathscr{B}\left(\mathbf{R}_{+}^{2}\right)$, то случайное поле $(z, \omega) \mapsto \int_{] 0, z]} \beta(z, u) \mu(z, d u)$ является $\mathscr{T} \mid \mathscr{B}(\mathbf{R})$-измеримьм.

\section{СПИСОК ЛИТЕРАТУРЫ}

1. Колодий Н.А. Некоторые свойства случайных полей, связанных со стохастическими интегралами по сильным мартингалам. - Зап. науч. сем. ПОМИ, 2004, т. 320 , c. $80-96$.

2. Doléans $C$. Intégrales stochastiques dépendant d'un paramètre. - Publ. Inst. Statist. Univ. Paris, 1967, 16, № 1, p. 23-33.

3. Stricker C., Yor M. Calcul stochastique dépendant d'un paramètre. - Z. Wahrscheinlichkeitstheor. verw. Geb., 1978, v. 45, № 2, p. 109-133.

4. Колодий Н.А. Интегралы по двупараметрическим сильным мартингальным ядрам и их применения. - Обозр. прикл. и промышл. матем., 2004, т. 11, в. 1, c. $120-121$.

5. Гущин A.A. К общей теории случайных полей на плоскости. - Успехи матем. наук, 1982, т. 37, № 6, с. 53-74.

6. Скороход A.B. Об измеримости случайных процессов. - Теория вероятн. и ее примен., 1980, т. 25, № 1, с. 140-142.

7. Гущин А.А., Мишура Ю. С. Неравенства Девиса и разложение Ганди для двупараметрических сильных мартингалов. - Теория вероятн. и матем. статист., 1990, № 42, c. 27-35. 
8. Ватанабэ C., Икэда Н. Стохастические дифференциальные уравнения и диффузионные процессы. М.: Наука, 1986, 448 с.

9. Dozzi M. Two-parameter stochastic processes. - Math. Res., 1991, v. 61, p. 17-43.

Поступила в редакцию 29.VII.2008

(c) 2011 г.

ЛЮЛЬКО Я.А.*

\title{
О РАСПРЕДЕЛЕНИИ ВРЕМЕНИ, ПРОВОДИМОГО МАРКОВСКОЙ ЦЕПЬЮ НА РАЗНЫХ УРОВНЯХ ДО МОМЕНТА ДОСТИЖЕНИЯ ФИКСИРОВАННОГО СОСТОЯНИЯ ${ }^{1)}$
}

\begin{abstract}
Данная работа посвящена исследованию вопроса о нахождении распределения времени пребывания однородной марковской цепи $Z=$ $\left(Z_{k}\right)_{k \geqslant 0}($ со счетным множеством состояний $E$ ) на разных уровнях фазового пространства до момента первого достижения фиксированного состояния $b \in E$.

Работа состоит из двух частей. В первой части с помощью строго марковского свойства показано, что в общем случае распределение времени пребывания будет геометрическим (с массой в нуле). В качестве примера рассмотрено скошенное случайное блуждание $S^{\alpha}=\left(S_{k}^{\alpha}\right)_{k \geqslant 0}$ с параметром $\alpha \in[0,1]$, для которого распределение времени пребывания найдено в явном виде.

Во второй части работы делается предельный переход от времени пребывания скошенного случайного блуждания к локальному времени скошенного броуновского движения $W^{\alpha}=\left(W_{t}^{\alpha}\right)_{t \geqslant 0}$. При этом основным инструментом для предельного перехода служит обобщенный принцип инвариантности Донскера-Прохорова.
\end{abstract}

Ключевые слова и фразы: марковская цепь, скошенное броуновское движение, локальное время, марковские моменты, принцип инвариантности Донскера-Прохорова.

\section{1. Введение.}

1.1. Пусть заданы вероятностное пространство $(\Omega, \mathscr{F}, \mathbf{P})$ и однородная марковская цепь $Z=\left(Z_{k}\right)_{k \geqslant 0}$ с множеством фазовых состояний $E=\left\{i_{0}, i_{1}, i_{2}, \ldots\right\}$, начальным распределением $p_{0}(i)=\mathbf{P}\left(Z_{0}=i\right)$ и переходными вероятностями $p_{i, j}=\mathbf{P}\left(Z_{n}=\right.$ $\left.j \mid Z_{n-1}=i\right), i, j \in E$. В качестве фильтрации рассмотрим естественную фильтрацию $\mathbb{F}=\left(\mathscr{F}_{n}\right)_{n \geqslant 0}, \mathscr{F}_{n}=\sigma\left(Z_{0}, \ldots, Z_{n}\right)$. Для каждого $x \in E$ определим меру $\mathbf{P}_{x}$ следующим образом: $\mathbf{P}_{x}(A)=\mathbf{P}\left(A \mid Z_{0}=x\right)$ для любого $A \in \mathscr{F}$. Положим

$$
N_{n}(a)=\sum_{k=0}^{n} \mathbb{I}\left(Z_{k}=a\right),
$$

где $a \in E$, и пусть $\tau_{b}=\inf \left\{k>0: Z_{k}=b\right\}$ для $b \in E$.

* Московский государственный университет им. М.В. Ломоносова, механикоматематический факультет, Ленинские горы, 119991 Москва, Россия; е-таil: yaroslav.lyulko@gmail.com

1) Работа выполнена при поддержке Фонда содействия отечественной науки. 